Article

\title{
Fluid-Structure Interaction in coronary Stents: a Discrete Multiphysics approach
}

\author{
Adamu Musa Mohammed ${ }^{1,2, *}$, Mostapha Ariane ${ }^{3}$ and Alessio Alexiadis ${ }^{*}$ \\ 1 School of Chemical Engineering, University of Birmingham, Birmingham B15 2TT, UK; amm702@bham.ac.uk, A.Alexiadis@bham.ac.uk \\ 2 Department of Chemical Engineering, Faculty of Engineering and Engineering Technology, Abubakar Tafawa Balewa University, Bauchi \\ 740272, Nigeria; ammohd@atbu.edu.ng \\ 3 Department of Materials and Engineering, Sayens-University of Burgundy, 21000 Dijon, France; Mostapha.Ariane@u-bourgogne.fr \\ * Correspondence: 1. amm702@bham.ac.uk or ammohd@atbu.edu.ng; Tel.: +44-(0)776-717-3356 \\ 2. A.Alexiadis@bham.ac.uk; Tel.: +44 (0) 1214145305
}

\begin{abstract}
Stenting is a common method for treating atherosclerosis. A metal or polymer stent is deployed to open the stenosed artery or vain. After the stent is deployed, the blood flow dynamics influences the mechanics by compressing and expanding the structure. If the stent does not respond properly to the resulting stress, vascular wall injury or re-stenosis can occur. In this work, a Discrete Multiphysics is used to study the mechanical deformation of the coronary stent and its relationship with the blood flow dynamics. The major parameters responsible for deforming the stent are sort in terms of dimensionless numbers and a relationship between the elastic forces in the stent and pressure forces in the fluid is established. The blood flow and the stiffness of the stent material contributes significantly to the stent deformation and affects the rate if deformation. The stress distribution in the stent is not uniform with the higher stresses occurring at the nodes of the structure.
\end{abstract}

Keywords: discrete multiphysics; smooth particle hydrodynamics; lattice Spring Model; fluid-structure interaction; particle-based method; coronary stent; mechanical deformation,

\section{Introduction}

Atherosclerosis and thrombosis are the terms used in describing a blockage (stenosis) of arteries due to plaque buildup or blood clotting. This blockage hinders the smooth transportation of blood through these arteries and consequently posed a great danger of possible severe coronary heart attack. Coronary artery which transport oxygenated blood to the heart often experience atherosclerosis and in an attempt to prevent such complication many studies were carried out on the types of plaque and its morphologies [1] that occurred in human arteries. It is paramount to manage or prevent such complications in order to restore normal blood flow. One of the ways of restoring the blood flow (revascularization) through the stenosed artery is stent implantation [2]. Coronary stents are tubular scaffolds that are deployed to recover the shrinking size of a diseased (narrowed) arterial segment [3] and stenting is a primary treatment of a stenosed artery that hinders smooth blood flow [4].

The stents used in clinical practice come in different geometry and design which implies varying stress distribution within local hemodynamic environment as well as on the plaque and artery $[5,6]$.The stent structure also induces different levels of Wall Shear Stress (WSS) on the wall of the artery [3,7]. Many cases of stent failure due to unbearable stress were reported and therefore, a careful study on how these stresses are distributed is needed. In fact, stent fracture or failure often occurs after stent implantation, and it can be avoidable if the mechanical property and the performance of the material is predicted. An ideal stent should provide good arterial support after expansion by having 
high radial strength. It should also cause minimal injury to the artery when expanded and should have high flexibility for easy manoeuvring during insertion [8,9].

Studies on the different stent designs and how it affect its mechanical performance were reported [6,7]. Stent deformation and fracture after implantation was also investigated [10-14]. Moreover, numerical modelling and simulations were also used in studying coronary stent and stent implantation. For instance, Di Venuta et al., 2017 carried out numerical simulation on a failed coronary stent implant on the degree of residual stenosis and discovered that the wall shear stress (WSS) increases monotonically, but not linearly with the degree of residual stenosis [2]. Simulation of hemodynamics in a stented coronary artery and for in-stent restenosis were performed by $[15,16]$.

With a few exceptions [17-19], the mechanical properties of the stent and the blood fluid dynamics around the stent were studied separately. In this work, we propose a Fluid Structure Interaction (FSI) model that calculates the stress on the stent produced by the pulsatile flow around the stent; both the stent mechanics and the blood hydrodynamics are calculated at the same time. The model is based on Discrete Multiphysics (DMP) [20], which has been used in a variety of FSI problems in biological systems such as the intestine [21], aortic valve [22,23], the lungs [22], deep venous valves [24,25]. In this study, therefore, we use DMP to develop a FSI 3D coronary stent model coupled with the blood hydrodynamics and analyse the mechanical deformations produced by the flow hydrodynamic.

\section{Methods}

\subsection{Discrete multiphysics}

Discrete Multiphysics combines together particle based techniques such as Smoother Particle Hydrodynamics (SPH) [26,27], Discrete Element Method (DEM) [28,29], Lattice Spring Model (LSM) [30,31] , Peridynamics (PD) [32] and even Artificial Neural Networks (ANN) [33,34]. In this case, the model couples SPH and LSM. The computational domain is divided into liquid domain and solid domain. The liquid domain represents the blood, and it is modelled with SPH particles; the solid domain represents the stent and the arterial walls and it is modelled with LSM particles. Details on SPH theory can be found in [35], and of LSM in [36,37]. Here a brief introduction of the equations used in SPH and LSM is provided.

\subsection{Smooth Particle Hydrodynamics (SPH)}

This section provides a basic introduction to SPH; additional details can be found in [35,38]. The general idea of SPH is to approximate a partial differential equation over a group of movable computational particles that are not connected over a grid or a mesh [31]. Newton's second law is integrated to give an approximate motion of the particles characterized by their own properties such as mass, velocity, pressure and density expressed by the fundamental identity:

$$
f(r)=f\left(r^{\prime}\right) \delta\left(r-r^{\prime}\right) d r^{\prime}
$$

where $f(r)$ is a generic function defined over the volume, $r$ is the position where the property is measured, and $\delta(r)$ is the delta function which is approximated by a smoothing (Kernel) function $W$ over a characteristic with $h$ (smoothing length). In this study, we use the so-called Lucy Kernel [35]. This approximation give rise to

$$
f(r) \approx f\left(r^{\prime}\right) W\left(r-r^{\prime}, h\right) d r^{\prime}
$$

which can be discretised over a series of particles of mass $m=\rho(r) \mathrm{d} r$ obtaining 


$$
f(r) \approx \sum_{i} \frac{m_{i}}{\rho_{i}} f\left(r_{i}\right) W\left(r-r_{i}, h\right)
$$

where $m_{i}$ and $\rho_{i}$ are the mass and density of the $i^{\text {th }}$ particles, and $i$ ranges over all particles withing the smoothing Kernel. Using this approximation, the Navier-Stoke equation can be discretised over a series of particles to obtain:

$$
m_{i} \frac{d v_{i}}{d t}=\sum_{j} m_{i} m_{j}\left(\frac{P_{i}}{\rho_{i}^{2}}+\frac{P_{j}}{\rho_{j}^{2}}+\Pi_{i, j}\right) \nabla_{j} W_{i, j}+f_{i}
$$

where $v$ is the particle velocity, $t$ the time, $m$ is the mass, $\rho$ the density and $P$ the pressure associated with particles $i$ and $j$. The term $f_{i}$ is the volumetric body force acting on the fluid and $\Pi_{i, j}$ introduces the viscous forced as defined by [39]. An equation of state is required to relates pressure and density. In this paper, Tait's equation of state is used:

$$
P(\rho)=\frac{c_{0} \rho_{0}}{7}\left[\left(\frac{\rho}{\rho_{0}}\right)^{7}-1\right]
$$

where $c_{0}$ and $\rho_{0}$ are a reference sound speed and density. To ensure weak compressibility, $c_{0}$ is chosen to be at least 10 times larger than the highest fluid velocity.

\subsection{Lattice Spring Model (LSM)}

Elastic objects can be simulated using lattice spring models. As already discussed in [23], the main element of this model composed of mass point and linear spring which exerts forces at the nodes connected by a linear spring and placed on a lattice Any material point of the body can be referred to by its position vector $r=(x, y, z)[40]$; when the body undergo deformation its position changes and the displacement is related to the applied force as:

$$
F=k\left(l-l_{0}\right)
$$

where $F$ is the force, $l_{0}$ is the initial distance between two particles, $l$ is the instantaneous distance, and $k$ the spring constant (or Hookean constant).

According to [36], in a regular cubic lattice structure, the spring constant is related to the bulk modulus of the material by

$$
K=\frac{5}{3} \frac{k}{l_{0}}
$$

and

$$
E=\frac{3}{2} K
$$

where $K$ is the bulk modulus, $E$ the young modulus, $l_{0}$ is the initial particle distance and $k$ the spring constant. From eq. 7 and 8 the spring constant is then related to the young modulus of the material by

$$
k=\frac{E l_{0}}{2.5}
$$

\section{Model and geometry}

A three-dimensional stent model including blood flow hydrodynamics and stent mechanics is developed. The model simulates the blood dynamics in 3D channel similar to a coronary artery with $1.5 \cdot 10^{-3} \mathrm{~m}$ internal radius, including a PS-shape stent of 4 struts in $x$-direction and 4 struts in the $z$-direction (circumference). The stent has a 
thickness of $100 \mu \mathrm{m}$, and 7.5mm length, the size that is within the range of the stent used in clinical practice [41]. We choose a PS-Shaped because it performs better compare to most commercially-shaped stents as reported by [19].

A script file and data file were written for the particle model and the simulations were run with LAMMPS, an open-source software [42]. The three-dimensional model consists of 1,862,804 particles: 1,609,452 particles for the fluid, 46,336 particles for the stent and 207,016 particles for the arterial wall. The fluid has a density $(\rho)$ of $1056 \mathrm{~kg} \mathrm{~m}^{-3}$ and viscosity $(\mu) 0.0035 \mathrm{~Pa} \cdot \mathrm{s}$. Figure 1 shows the section geometry of the stent within and outside the arterial wall. Local acceleration term $g_{0}$ was included to force the fluid to flow at a particular velocity. The inclusion of the local velocity is due to the unsteady or pulsatile flow existing in the cardiovascular system [43]. Womersley parameter $\alpha$, which is the ratio of unsteady force to viscous force, was used in the model to induce the velocity profile of the flow. The particle spacing is $3.33 \cdot 10^{-5} \mathrm{~m}$. The optimal spacing value is obtained after several simulations with different particle spacings to make sure the results are independent of the particle resolution. Stress and deformation and other postprocessing calculations were done with the open-source software OVITO [44]. The arterial wall is assumed to be rigid with no-slip condition [19] whereas the stent is elastic. Figure 1a and b show a section of the solid geometry which include the stent and the wall.
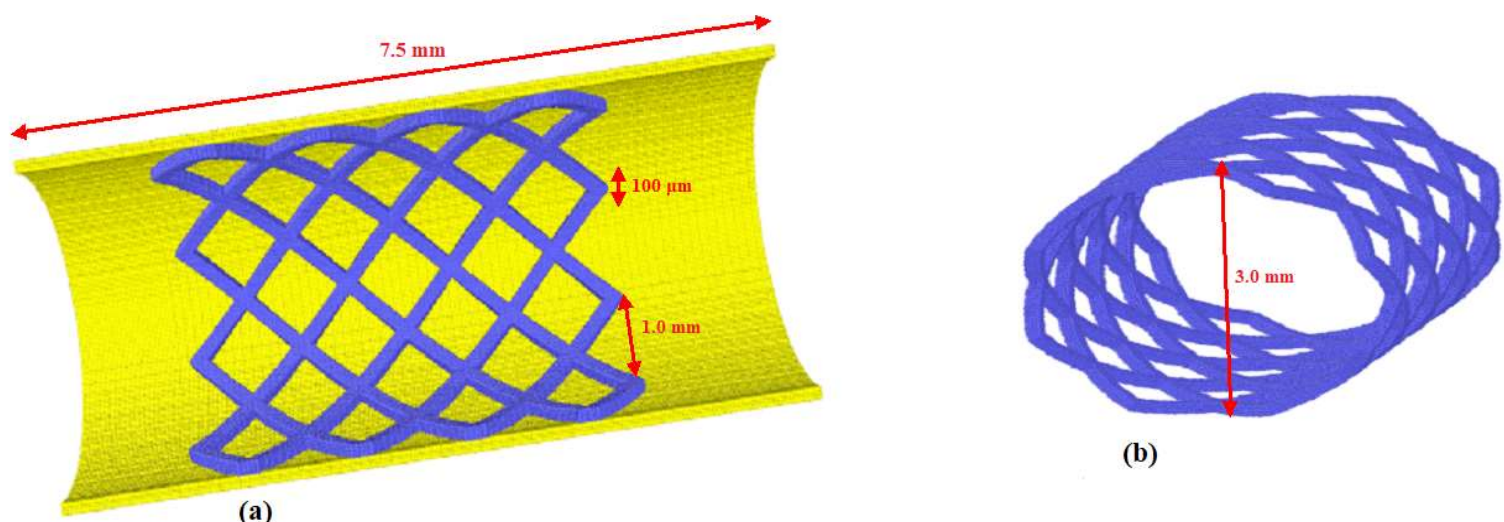

(b)

Figure 1. Illustration of the 3D stent geometry at different viewpoints.

To better understand the hydrodynamics of the fluid and to be able to access the deformation of the stent, the discussion is carried out in terms of dimensionless numbers. According to the Buckingham $\pi$ theorem, a physically meaningful equation involving $n$ physical variables can be rewritten in terms of a set of $p=n-k$ dimensionless parameters $\Pi_{1}, \Pi_{2}, \ldots, \Pi_{p}$, where $k$ is the number of physical dimensions involved. In this case, the physiochemical properties of blood are constant, and the geometry is fixed. Therefore, we want to express the resulting stress on the stent as a function $f$ of the type

$$
\sigma=f(P, h, E)
$$

where $\mathrm{s}\left[\mathrm{kg} \mathrm{m}^{-1} \mathrm{~s}^{-2}\right]$ is the stress on the stent, $P\left[\mathrm{~kg} \mathrm{~m}^{-1} \mathrm{~s}^{-2}\right]$ the dynamic pressure in the fluid (the force exerted by the fluid to the stent depends on $P$ ), $d[\mathrm{~m}]$ a characteristic length of the stent (here, we use the thickness of the stent) and $E[\mathrm{~kg}$ $\mathrm{m}^{-1} \mathrm{~s}^{-2}$ ] the Young Modulus.

In this case, the dynamic pressure can be written in terms of fluid average velocity $v$ and density $\mathrm{r}$,

$$
P=\rho v^{2}
$$

Moreover, $k\left[\mathrm{~kg} \mathrm{~s}^{-2}\right]$ and $E$ are related in eq.9. Therefore, assuming that the lattice spacing is fixed, we can replace eq. (10) with

$$
\sigma=f(\rho, v, d, k)
$$


Since we have 5 variables and 3 units, we can rewrite eq. 12 based on two dimensionless numbers

$$
\Pi_{2}=\varphi\left(\Pi_{1}\right)
$$

The first dimensionless number can be defined as

$$
\Pi_{1}=\frac{k}{\rho v^{2} h} \frac{\text { [elastic forces that contrast deformation (in the solid)] }}{\text { [pressure forces that tend to deform the stent (from the liquid)] }}
$$

Knowing the typical ranges of $E$ and $d$ for the stent, and $\rho$ and $v$ for the blood, we can calculate the typical range of $\Pi_{1}$. The second parameter $\Pi_{2}$ can be defined as

$$
\Pi_{2}=\frac{\sigma d}{k}
$$

We can have different types of $\Pi_{2}$ according to the type of stress we use in eq. 15. The stress tensor has 6 independent components that can be composed in different ways to provide different types of information. One possibility is to us the Frobenius norm.

$$
\sigma^{F}=\sqrt{\sigma_{x x}^{2}+\sigma_{y y}^{2}+\sigma_{z z}^{2}+2 \sigma_{x y}^{2}+2 \sigma_{x z}^{2}+2 \sigma_{y z}^{2}}
$$

In this case, we have a $\Pi_{2}$ based on the Frobenius norm

$$
\Pi_{2}^{F}=\frac{\sigma^{F} d}{k}
$$

that expresses, in dimensionless form, the total stress in the stent. Another possibility is the von Mises stress

$$
\sigma^{V}=\sqrt{\frac{1}{2}\left[\left(\sigma_{x x}-\sigma_{y y}\right)^{2}+\left(\sigma_{y y}-\sigma_{z z}\right)^{2}+\left(\sigma_{z z}-\sigma_{y y}\right)^{2}\right]+3\left(\sigma_{x y}^{2}+\sigma_{y z}^{2}+\sigma_{z x}^{2}\right)}
$$

which provides another $\Pi_{2}$ number defined as

$$
\Pi_{2}^{V}=\frac{\sigma^{V} d}{k}
$$

Physically, $\Pi_{2}$, and $\Pi_{2}$ are dimensionless stresses and can have both a local form $\Pi_{2}(x, y, z)$ (when we calculate them at each $x, y, z$ position), and a global form $\left\langle\Pi_{2}\right\rangle$ (when we average them over the whole stent).

Table1. Parameters needed for the simulation.

$\mathrm{SPH}$

\begin{tabular}{ll}
\hline Number of SPH fluid particle & $1,609,452$ \\
Mass of each particle (fluid) & $3.41 \times 10^{-12} \mathrm{~kg}$ \\
Length $L$ & $7.5 \times 10^{-3} \mathrm{~m}$
\end{tabular}




\begin{tabular}{ll} 
Diameter $D$ & $3.0 \times 10^{-3} \mathrm{~m}$ \\
Particle spacing $l$ & $3.33 \times 10^{-5} \mathrm{~m}$ \\
Smoothing length $h$ & $7.5 \times 10^{-5} \mathrm{~m}$ \\
Local acceleration term $g_{0}$ & $0.47138-1.25 \mathrm{~m} \mathrm{~s}^{-2}$ \\
Fluid Density $\rho$ & $1056 \mathrm{~kg} \mathrm{~m}^{-3}$ \\
Viscosity $\mu$ & $0.0035 \mathrm{~Pa} \cdot \mathrm{s}$ \\
Sound speed $c_{0}$ & $4 \mathrm{~m} \mathrm{~s}^{-1}$ \\
Alpha $\alpha$ & $0.1-0.25[-]$ \\
Time step $\Delta t$ & $1 \times 10^{-7} \mathrm{~s}$ \\
\hline & \\
\hline Number of SPH stent & LSM \\
Number of SPH wall particle & 46,336 \\
Mass of each particle of the stent (Solid) & 207,016 \\
Mass of each particle of the wall (Solid) & $3.41 \times 10^{-12} \mathrm{~kg}$ \\
Stent thickness $d$ & $6.0 \times 10^{-12} \mathrm{~kg}$ \\
Elastic constant $\mathrm{k}$ & $1.0 \times 10^{-4} \mathrm{~m}$ \\
\hline
\end{tabular}

\section{Results and Discussion}

Three flow velocities of $0.4 \mathrm{~ms}^{-1}, 0.23 \mathrm{~ms}^{-1}$ and $0.16 \mathrm{~ms}^{-1}$ were chosen to represent the normal coronary artery and the baseline flow, respectively. The blood flow velocity observed within the stent ranges from $0.23 \mathrm{~ms}^{-1}$ to $0.4 \mathrm{~ms}^{-1}$, whereas the minimum flow velocity which may occur due to stenosis is $0.16 \mathrm{~ms}^{-1}$ and taken to be the baseline [45]. Velocity profile at different viewpoints is shown in Figure 2.

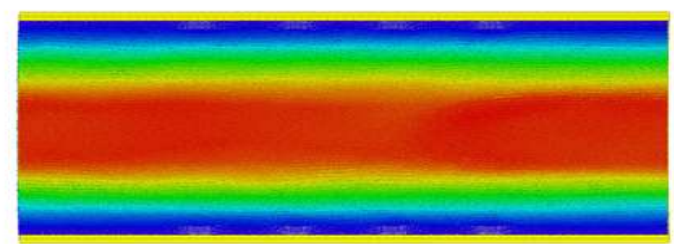

(a)
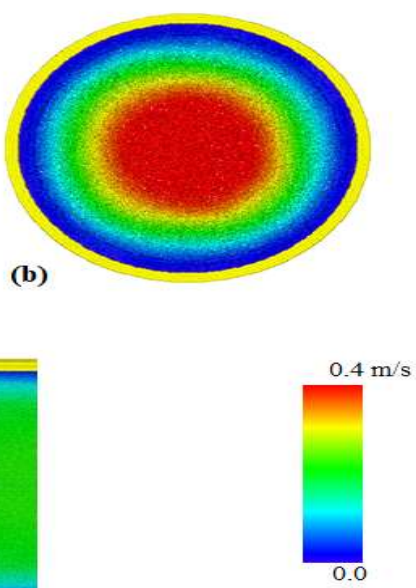

(c)

Figure 2. Velocity profile; (a) $x-y$ view (steady state profile), (b) y-z view, and (c) parabolic profile at the beginning of the flow 


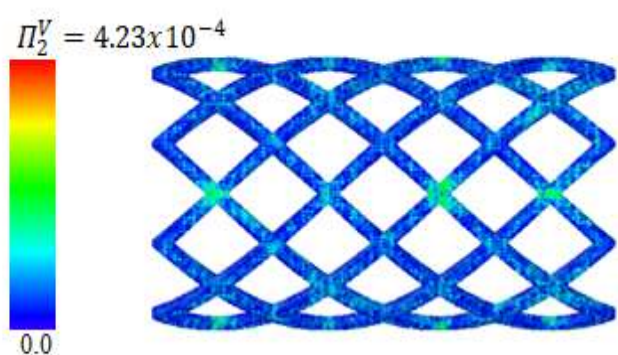

(a) $\Pi_{1}=296$

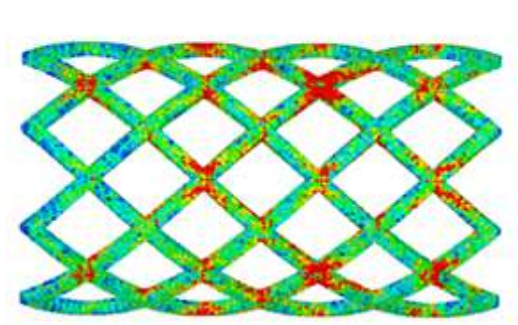

(b) $\quad \Pi_{1}=1480$

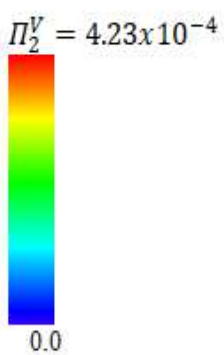

Figure 3. Local $\Pi^{v_{2}}$ at different values of $\Pi_{1}$

The dimensionless von Mises stress is shown in Figure 3 for two stents at different $\left\langle\Pi_{1}\right\rangle$. Different value of $\left\langle\Pi_{1}\right\rangle$ means different $k$ and $v$. It is shown that the stress is more severe at the nodes (joints) with higher stress at higher $\left\langle\Pi_{1}\right\rangle$ as clearly shown in Figure $3 \mathrm{~b}$. This may lead to potential stent failure (rapture) at the joins or size change of the stent resulting from compression or expansion. The expansion characteristics of a stent is the main causes for vascular wall injuries [46]. Either of these conditions (failure or size change) will cause severe pain and damage to the patient and lead to restenosis and or stent redeployment.

\section{Frobenius}

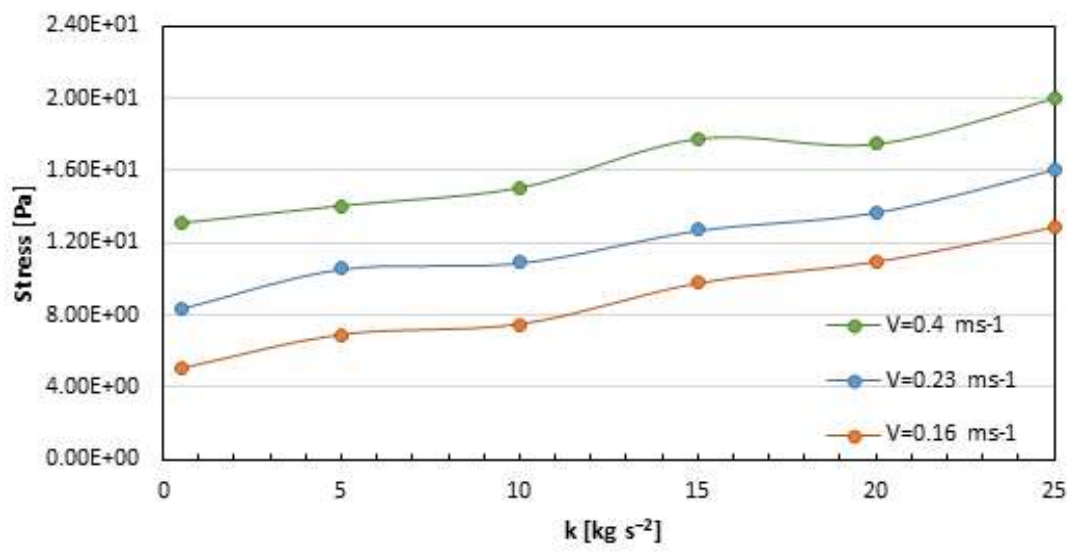

Figure 4. Stress (Frobenius norm of the stress tensor) with respect to $k$ and $v$

The result is first presented in Figure 4 and shows how the stress varies with $k$ and $v$. This is then sorted in dimensionless form and presented in Figure 5 and Figure 6, which shows the average stress $\left\langle\Pi_{2}{ }_{2}\right\rangle$, and $\left\langle\Pi v_{2}\right\rangle$ versus $<\Pi_{1}>$ in dimensionless form. If we use dimensionless numbers the three curves of Figure 4 collapse in only one curve (Figure 5 and Figure 6). 


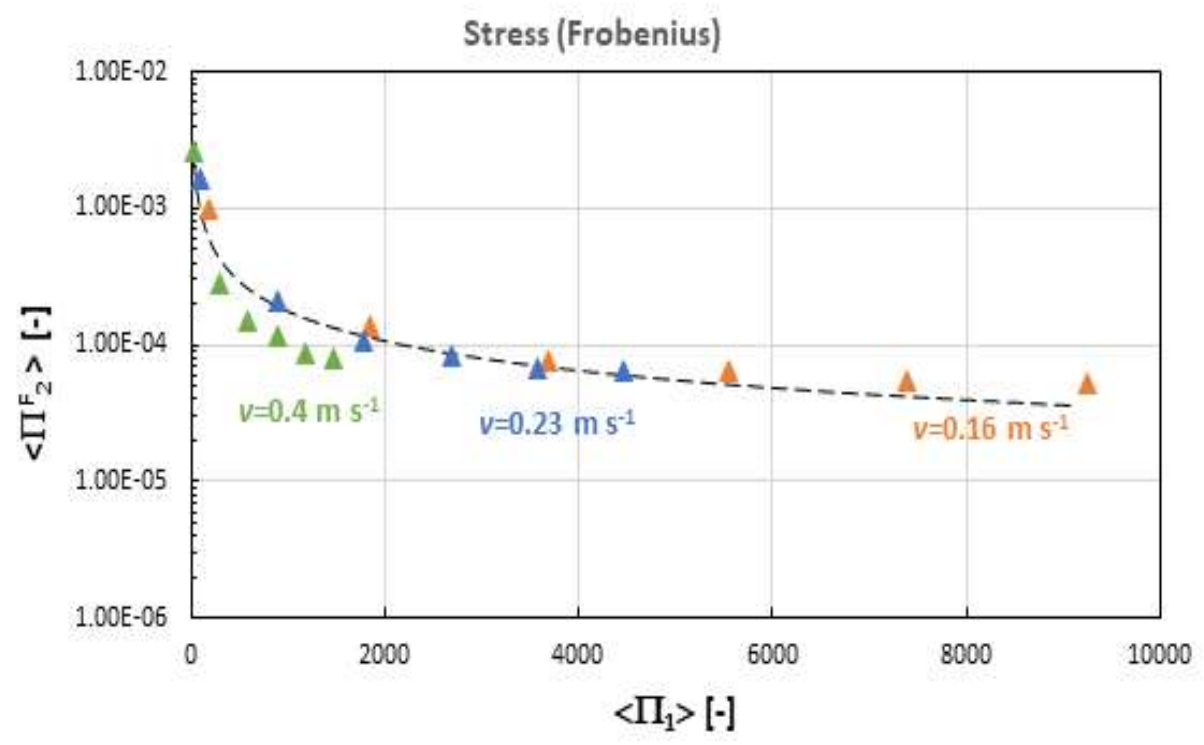

Figure 5. Relationship between average stress $\left\langle\Pi^{F_{2}}>\right.$ and $\left\langle\Pi_{1}>\right.$

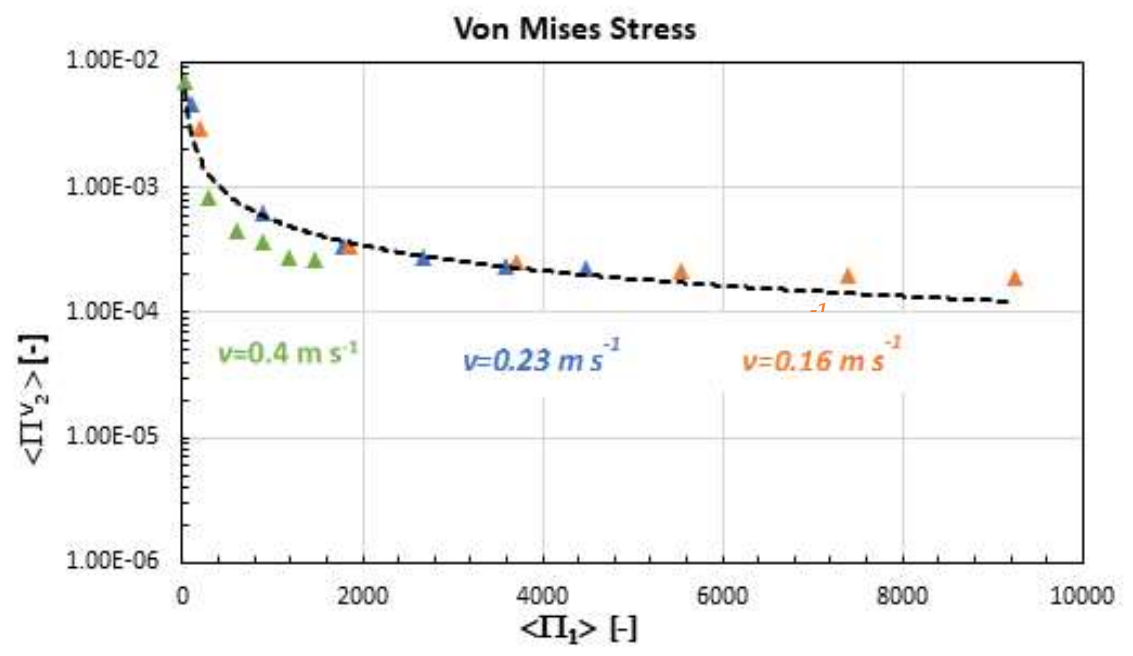

Figure 6. Relationship between average stress $\left\langle\Pi_{2} v_{2}\right.$ and $\left\langle\Pi_{1}>\right.$

The plot confirms that the stress can be effectively sort out with two dimensionless numbers based on the Buckingham $\pi$ theorem. The three curves of Figure 4 can be fit by the same function as indicated in eq. 13 . This function can be approximated by the following correlation (dotted line in)

$$
<\Pi_{2}^{F}>=0.026\left\langle\Pi_{1}\right\rangle^{-0.723}
$$

The same thing can be done for the Von Mises stress which also has a correlation

$$
<\Pi_{2}^{V}>=0.058\left\langle\Pi_{1}\right\rangle^{-0.6737}
$$

Numerically, we identified that the dimensionless numbers computed can be used as the fundamental group of the system in which the stress can be express in term of $\Pi_{1}$ and $\Pi_{2}$. 


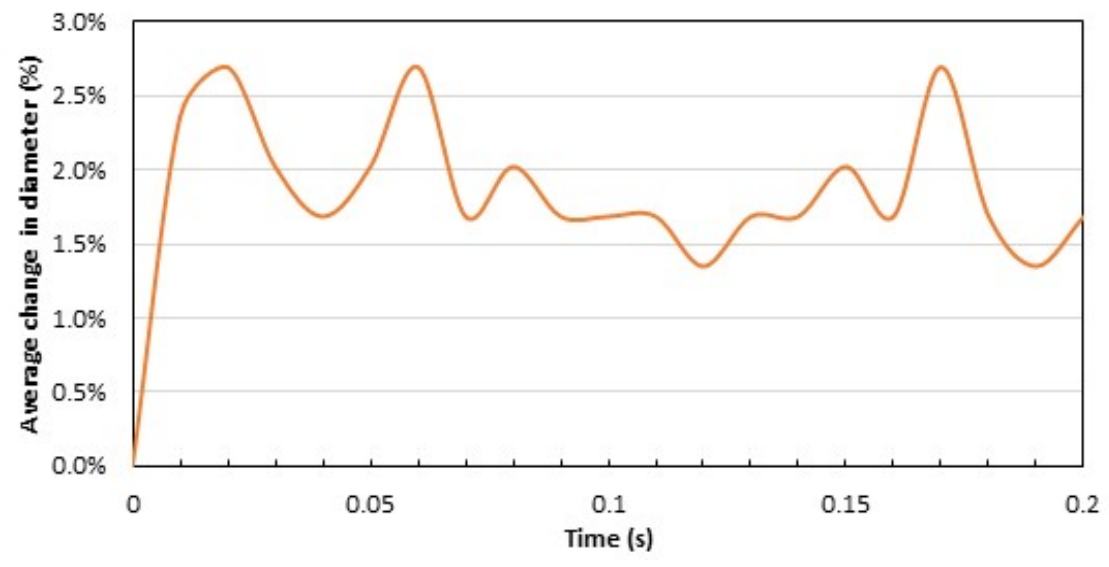

Figure 7. Average diameter change with time.

Because of the pulsatile flow, the stent contracts and expands during the simulation. This causes the diameter of the stent to change with the flow. Figure 7 shows that the percentage of stent diameter change is also pulsatile. Arterial blood flow is pulsatile in nature $[47,48]$, therefore, it is expected that the step time deformation will also be pulsatile. The deformation is not only a function of the pressure forces from the liquid (blood) but also the elastic forces from the solid (stent). For that reason, several oscillations modes occur at the same time and the diameter change is not a simple repetition of the pulsatile flow.

Another incidence of likely vascular wall injury associated with stent expansion which can be quantified using the model is the so-called dogboning (DB) effect/ratio. This phenomenon occurs when the stent expands at the ends, resulting into increased stress and injuries at the arterial wall [46]. This occurs when the diameter expands at both ends of the stent and contracts at the centre. The dogboning ratio is defined as,

$$
D B=\frac{D_{\text {max }, \text { end }}-D_{\text {min, } \text { central }}}{D_{\text {max }, \text { end }}} \times 100 \%
$$

where $D_{\max \text {,end }}$ is the maximum stent diameter at the end (distal and proximal), and $D_{\text {min,central }}$ is the minimum stent diameter at the centre. Our model is capable of analysing the dogboning ratio which could be used to assess and reduce the potential risk of the vascular wall injury, and therefore, in this study, DB was calculated to be $4.4 \%$. This is less than the $6.3 \%$ reported by [19] for PS-shaped stent.

\section{Conclusions}

In this paper, a Discrete Multiphysics model is developed to simulate a coronary stent accounting for both its hemodynamics and mechanical stress. The model is three-dimensional and includes both the fluid (blood) and the solid structures (arterial wall and stent) and it is used to study the link between the flow dynamics and the mechanical deformation of the stent. The mechanical stress is computed using dimensionless numbers and a relationship between elastic forces and pressure forces was established. The results show that the blood flow contributes significantly to the stent deformation and the stiffness of the stent material affected the rate of deformation. Nonuniform stress distributions are observed. In particular, high stresses are observed at the nodes of the stent. Therefore, the model can be used to predict the deformation of the stent once in place and the conditions that can potentially cause its failure. 
Author Contributions: Conceptualization, M.A., A.M.M., A.A; simulation and visualization, A.M.M.; numerical calculations, A.M.M., M.A. and A.A; interpretation and analysis of results, A.M.M. and A.A; writing-original draft preparation, A.M.M.; supervision, A.A. and M.A.; writing - review and editing, A.A., A.M.M., and M.A.; input script, M.A. and A.M.M. All authors have read and agreed to the published version of the manuscript.

Funding: This research received no external funding.

Acknowledgments: The Nigerian Petroleum Technology Development Fund (PTDF) is acknowledged for the provision of a scholarship to Adamu Musa Mohammed.

Conflicts of Interest: The authors declare no conflict of interest.

\section{References}

1. Otsuka, F.; Yasuda, S.; Noguchi, T.; Ishibashi-Ueda, H. Pathology of Coronary Atherosclerosis and Thrombosis. Cardiovasc. Diagn. Ther. 2016, 6, 396-408, doi:10.21037/cdt.2016.06.01.

2. Di Venuta, I.; Boghi, A.; Gori, F. Three-Dimensional Numerical Simulation of a Failed Coronary Stent Implant at Different Degrees of Residual Stenosis. Part I: Fluid Dynamics and Shear Stress on the Vascular Wall. Numerical Heat Transfer, Part A: Applications 2017, 71, 638-652, doi:10.1080/10407782.2017.1293985.

3. Pant, S.; Bressloff, N.W.; Limbert, G. Geometry Parameterization and Multidisciplinary Constrained Optimization of Coronary Stents. Biomech Model Mechanobiol 2012, 11, 61-82, doi:10.1007/s10237-011-0293-3.

4. Hsiao, H.-M.; Lee, K.-H.; Liao, Y.-C.; Cheng, Y.-C. Hemodynamic Simulation of Intra-Stent Blood Flow. Procedia Engineering 2012, 36, 128-136, doi:10.1016/j.proeng.2012.03.020.

5. Wei, L.; Chen, Q.; Li, Z. Influences of Plaque Eccentricity and Composition on the Stent-Plaque-Artery Interaction during Stent Implantation. Biomech Model Mechanobiol 2019, 18, 45-56, doi:10.1007/s10237-018-1066-z.

6. Colombo, A.; Stankovic, G.; Moses, J.W. Selection of Coronary Stents. Journal of the American College of Cardiology 2002, 40, 1021-1033, doi:10.1016/S0735-1097(02)02123-X.

7. Balossino, R.; Gervaso, F.; Migliavacca, F.; Dubini, G. Effects of Different Stent Designs on Local Hemodynamics in Stented Arteries. Journal of Biomechanics 2008, 41, 1053-1061, doi:10.1016/j.jbiomech.2007.12.005.

8. Duraiswamy, N.; Jayachandran, B.; Byrne, J.; Moore, J.E.; Schoephoerster, R.T. Spatial Distribution of Platelet Deposition in Stented Arterial Models Under Physiologic Flow. Ann Biomed Eng 2005, 33, 1767-1777, doi:10.1007/s10439-005-7598-2.

9. Pant, S.; Bressloff, N.W.; Forrester, A.I.J.; Curzen, N. The Influence of Strut-Connectors in Stented Vessels: A Comparison of Pulsatile Flow Through Five Coronary Stents. Ann Biomed Eng 2010, 38, 1893-1907, doi:10.1007/s10439-010-9962-0.

10. Finet, G.; Rioufol, G. Coronary Stent Longitudinal Deformation by Compression: Is This a New Global Stent Failure, a Specific Failure of a Particular Stent Design or Simply an Angiographic Detection of an Exceptional PCI Complication? EuroIntervention 2012, 8, 177-181, doi:10.4244/EIJV8I2A29.

11. Choudhury, T.R.; Al-Saigh, S.; Burley, S.; Li, L.; Shakhshir, N.; Mirhosseini, N.; Wang, T.; Arnous, S.; Khan, M.A.; Mamas, M.A.; et al. Longitudinal Deformation Bench Testing Using a Coronary Artery Model: A New Standard? Open Heart 2017, 4, e000537, doi:10.1136/openhrt-2016-000537.

12. Ding, H.; Zhang, Y.; Liu, Y.; Shi, C.; Nie, Z.; Liu, H.; Gu, Y. Analysis of Vascular Mechanical Characteristics after Coronary Degradable Stent Implantation. BioMed Research International 2019, 2019, 1-9, doi:10.1155/2019/8265374.

13. Chinikar, M.; Sadeghipour, P. Coronary Stent Fracture: A Recently Appreciated Phenomenon with Clinical Relevance. CCR 2014, 10, 349-354, doi:10.2174/1573403X10666140404105923.

14. Alqahtani, A.; suwaidi, J.; Mohsen, M. Stent Fracture: How Frequently Is It Recognized? Heart Views 2013, 14, 72, doi:10.4103/1995-705X.115501. 
15. Faik, I.; Mongrain, R.; Leask, R.L.; Rodes-Cabau, J.; Larose, E.; Bertrand, O. Time-Dependent 3D Simulations of the Hemodynamics in a Stented Coronary Artery. Biomed. Mater. 2007, 2, S28-S37, doi:10.1088/1748-6041/2/1/S05.

16. Caiazzo, A.; Evans, D.; Falcone, J.-L.; Hegewald, J.; Lorenz, E.; Stahl, B.; Wang, D.; Bernsdorf, J.; Chopard, B.; Gunn, J.; et al. A Complex Automata Approach for In-Stent Restenosis: Two-Dimensional Multiscale Modelling and Simulations. Journal of Computational Science 2011, 2, 9-17, doi:10.1016/j.jocs.2010.09.002.

17. Beier, S.; Ormiston, J.; Webster, M.; Cater, J.; Norris, S.; Medrano-Gracia, P.; Young, A.; Cowan, B. Hemodynamics in Idealized Stented Coronary Arteries: Important Stent Design Considerations. Ann Biomed Eng 2016, 44, 315-329, doi:10.1007/s10439-015-1387-3.

18. Xu, J.; Yang, J.; Huang, N.; Uhl, C.; Zhou, Y.; Liu, Y. Mechanical Response of Cardiovascular Stents under Vascular Dynamic Bending. BioMed Eng OnLine 2016, 15, 21, doi:10.1186/s12938-016-0135-8.

19. Wei, L.; Leo, H.L.; Chen, Q.; Li, Z. Structural and Hemodynamic Analyses of Different Stent Structures in Curved and Stenotic Coronary Artery. Front. Bioeng. Biotechnol. 2019, 7, 366, doi:10.3389/fbioe.2019.00366.

20. Alexiadis, A. A Smoothed Particle Hydrodynamics and Coarse-Grained Molecular Dynamics Hybrid Technique for Modelling Elastic Particles and Breakable Capsules under Various Flow Conditions: SPH-CGMD HYBRID. Int. J. Numer. Meth. Engng 2014, 100, 713-719, doi:10.1002/nme.4782.

21. Schütt, M.; Stamatopoulos, K.; Simmons, M.J.H.; Batchelor, H.K.; Alexiadis, A. Modelling and Simulation of the Hydrodynamics and Mixing Profiles in the Human Proximal Colon Using Discrete Multiphysics. Computers in Biology and Medicine 2020, 121, 103819, doi:10.1016/j.compbiomed.2020.103819.

22. Ariane, M.; Kassinos, S.; Velaga, S.; Alexiadis, A. Discrete Multi-Physics Simulations of Diffusive and Convective Mass Transfer in Boundary Layers Containing Motile Cilia in Lungs. Computers in Biology and Medicine 2018, 95, 34-42, doi:10.1016/j.compbiomed.2018.01.010.

23. Mohammed, A.M.; Ariane, M.; Alexiadis, A. Using Discrete Multiphysics Modelling to Assess the Effect of Calcification on Hemodynamic and Mechanical Deformation of Aortic Valve. ChemEngineering 2020, 4, 48, doi:10.3390/chemengineering4030048.

24. Ariane, M.; Wen, W.; Vigolo, D.; Brill, A.; Nash, F.G.B.; Barigou, M.; Alexiadis, A. Modelling and Simulation of Flow and Agglomeration in Deep Veins Valves Using Discrete Multi Physics. Computers in Biology and Medicine 2017, 89, 96-103, doi:10.1016/j.compbiomed.2017.07.020.

25. Ariane, M.; Vigolo, D.; Brill, A.; Nash, F.G.B.; Barigou, M.; Alexiadis, A. Using Discrete Multi-Physics for Studying the Dynamics of Emboli in Flexible Venous Valves. Computers $\mathcal{E}$ Fluids 2018, 166, 57-63, doi:10.1016/j.compfluid.2018.01.037.

26. Albano, A.; Alexiadis, A. A Smoothed Particle Hydrodynamics Study of the Collapse for a Cylindrical Cavity. PLoS ONE 2020, 15, e0239830, doi:10.1371/journal.pone.0239830.

27. Albano, A.; Alexiadis, A. Non-Symmetrical Collapse of an Empty Cylindrical Cavity Studied with Smoothed Particle Hydrodynamics. Applied Sciences 2021, 11, 3500, doi:10.3390/app11083500.

28. Liu, W.; Wu, C.-Y. Modelling Complex Particle-Fluid Flow with a Discrete Element Method Coupled with Lattice Boltzmann Methods (DEM-LBM). ChemEngineering 2020, 4, 55, doi:10.3390/chemengineering4040055.

29. Ng, K.C.; Alexiadis, A.; Chen, H.; Sheu, T.W.H. A Coupled Smoothed Particle Hydrodynamics-Volume Compensated Particle Method (SPH-VCPM) for Fluid Structure Interaction (FSI) Modelling. Ocean Engineering 2020, 218, 107923, doi:10.1016/j.oceaneng.2020.107923.

30. Sahputra, I.H.; Alexiadis, A.; Adams, M.J. A Coarse Grained Model for Viscoelastic Solids in Discrete Multiphysics Simulations. ChemEngineering 2020, 4, 30, doi:10.3390/chemengineering4020030.

31. Ruiz-Riancho, I.N.; Alexiadis, A.; Zhang, Z.; Garcia Hernandez, A. A Discrete Multi-Physics Model to Simulate Fluid Structure Interaction and Breakage of Capsules Filled with Liquid under Coaxial Load. Processes 2021, 9, 354, doi:10.3390/pr9020354. 
32. Sanfilippo, D.; Ghiassi, B.; Alexiadis, A.; Hernandez, A.G. Combined Peridynamics and Discrete Multiphysics to Study the Effects of Air Voids and Freeze-Thaw on the Mechanical Properties of Asphalt. Materials 2021, 14, 1579, doi:10.3390/ma14071579.

33. Alexiadis, A. Deep Multiphysics and Particle-Neuron Duality: A Computational Framework Coupling (Discrete) Multiphysics and Deep Learning. Applied Sciences 2019, 9, 5369, doi:10.3390/app9245369.

34. Alexiadis, A.; Simmons, M.J.H.; Stamatopoulos, K.; Batchelor, H.K.; Moulitsas, I. The Duality between Particle Methods and Artificial Neural Networks. Sci Rep 2020, 10, 16247, doi:10.1038/s41598-020-73329-0.

35. Liu, G.R.; Liu, M.B. Smoothed Particle Hydrodynamics: A Meshfree Particle Method; World Scientific: New Jersey, 2003; ISBN 978-981-238-456-0.

36. Kot, M.; Nagahashi, H.; Szymczak, P. Elastic Moduli of Simple Mass Spring Models. Vis Comput 2015, 31, 13391350, doi:10.1007/s00371-014-1015-5.

37. Kot, M. Mass Spring Models of Amorphous Solids. ChemEngineering 2021, 5, 3, doi:10.3390/chemengineering5010003.

38. Monaghan, J.J. Smoothed Particle Hydrodynamics. Annu. Rev. Astron. Astrophys. 1992, 30, 543-574, doi:10.1146/annurev.aa.30.090192.002551.

39. Morris, J.P.; Fox, P.J.; Zhu, Y. Modeling Low Reynolds Number Incompressible Flows Using SPH. Journal of Computational Physics 1997, 136, 214-226, doi:10.1006/jcph.1997.5776.

40. Pazdniakou, A.; Adler, P.M. Lattice Spring Models. Transp Porous Med 2012, 93, 243-262, doi:10.1007/s11242-0129955-6.

41. Functionalized Cardiovascular Stents; Wall, J.G., Podbielska, H., Wawrzyńska, M., Eds.; Woodhead Publishing series in biomaterials; Elsevier, Woodhead Publishing: Duxford, United Kingdom ; Cambridge, MA, 2018; ISBN 978-008-100496-8.

42. Plimpton, S. Fast Parallel Algorithms for Short-Range Molecular Dynamics. Journal of Computational Physics 1995, 117, 1-19, doi:10.1006/jcph.1995.1039.

43. Ku, D.N. BLOOD FLOW IN ARTERIES. Annu. Rev. Fluid Mech. 1997, 29, 399-434, doi:10.1146/annurev.fluid.29.1.399.

44. Stukowski, A. Visualization and Analysis of Atomistic Simulation Data with OVITO-the Open Visualization Tool. Modelling Simul. Mater. Sci. Eng. 2010, 18, 015012, doi:10.1088/0965-0393/18/1/015012.

45. Vrints, C.J.; Claeys, M.J.; Bosmans, J.; Conraads, V.; Snoeck, J.P. Effect of Stenting on Coronary Flow Velocity Reserve: Comparison of Coil and Tubular Stents. Heart 1999, 82, 465-470, doi:10.1136/hrt.82.4.465.

46. Wiesent, L.; Schultheiß, U.; Schmid, C.; Schratzenstaller, T.; Nonn, A. Experimentally Validated Simulation of Coronary Stents Considering Different Dogboning Ratios and Asymmetric Stent Positioning. PLoS ONE 2019, 14, e0224026, doi:10.1371/journal.pone.0224026.

47. Huo, Y.; Kassab, G.S. Pulsatile Blood Flow in the Entire Coronary Arterial Tree: Theory and Experiment. American Journal of Physiology-Heart and Circulatory Physiology 2006, 291, H1074-H1087, doi:10.1152/ajpheart.00200.2006.

48. Cheung, Y. Systemic Circulation. In Paediatric Cardiology; Elsevier, 2010; pp. 91-116 ISBN 978-0-7020-3064-2. 